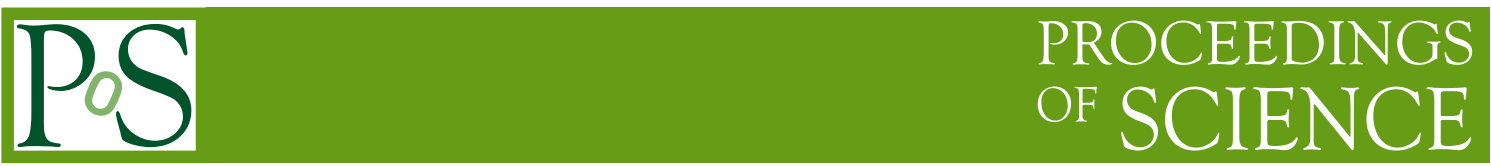

\title{
SModelS - new developments and applications
}

\author{
Ursula Laa*† \\ School of Physics and Astronomy \& School of Econometrics and Business Statistics, Monash \\ University, Melbourne VIC-3800 \\ E-mail: ursula.laa@monash.edu
}

\begin{abstract}
ATLAS and CMS have performed a large number of searches for physics beyond the Standard Model (BSM). The results are typically presented in the context of simplified models, containing only a few new particles with fixed decay branching ratios, and yielding generic upper limits on the cross section as a function of particle masses. The interpretation of these limits within realistic BSM scenarios is non-trivial and is best done by automated computational tools. SModelS is such an automatised tool, allowing to decompose models of new physics obeying a $\mathbb{Z}_{2}$ symmetry into simplified model components, and to compare these against a large database of experimental results. A recent release, version 1.1, extended the functionality from comparing to upper limit maps to using also efficiency maps (thus enabling the combination of simplified models), and includes likelihood and chi-square calculations, extended information on the topology coverage as well as an extended database of experimental results. This talk presented the tool emphasising in particular the new developments and discussed an example physics application, a recent study on the coverage of the pMSSM by the available simplified model results. This discussion illustrates how SModelS can be used to identify important constraints, untested regions and interesting new signatures. An outlook to future developments was also given.
\end{abstract}

ICHEP 2018, International conference on High Energy Physics

4-11 July 2018

Seoul, Korea

\footnotetext{
* Speaker.

${ }^{\dagger}$ For the SModelS collaboration.
} 


\section{Introduction}

Experimental searches for supersymmetry (SUSY) are typically interpreted in the context of simplified models, containing a limited set of new particles with fixed decay modes, allowing interpretation without large parameter spaces but also without introducing large model dependence. The generality and wide availability of these interpretations makes them an ideal input for the reinterpretation of the results in more complex models, thus providing a fast but conservative alternative to classic reinterpretation methods based on event and detector simulations. Fast because the simulation step has already been performed and all detector effects have been folded into the simplified model interpretation. Conservative because often only part of the total signal can be constrained, limited by the availability of results for topologies appearing in the model. Here we present the public tool SModelS that automates the comparison of generic model predictions against simplified model results.

\section{SModelS}

SModelS [1, 2, 3] provides a three-step procedure to implement this comparison. The first step consists in "decomposing" the generic model, i.e. given the tables of production cross sections and decay branching ratios (in SLHA format), SModelS constructs all relevant simplified model topologies, keeping track of the relevant mass parameters, and calculating the "weight" $=\sigma \times \mathrm{BR}$. This decomposition procedure is generic and works for any model with a $\mathbb{Z}_{2}$, by mapping onto a generalised description of simplified models. The result is a set of minimal topologies, that can be combined in a second step, according to what has been considered by the experiments. For example topologies with electrons and muons in the final state may be combined to constrain decay into leptons. The final step is then to compare the theory predictions, i.e. the weights for each topology to the experimental limits. For this step SModelS provides two different methods. The first one is using upper limit (UL) maps provided by the experiments. These maps contain limits on the weight with all detector effects folded in, as a function of the particle mass. Therefore they can directly be compared to the prediction, on a topology-by-topology basis. The second method is instead using efficiency maps $(E M)^{1}$, which instead encode the total selection efficiency as a function of masses. The EMs can thus be used to calculate the total visible cross section as the product of the weight and the efficiency. In this case different topologies contributing to the visible signal in the same signal region can be combined, and the sum of their visible cross sections can be compared to a corresponding limit evaluated based on the experimental result. EMs are often provided by the experiments, in addition they may also be puslished as results of phenomenological studies.

The main advantage of using SModelS is the fast comparison against a large database of experimental results. In addition SModelS is an efficient tool for the identification of the most relevant search channels and analysis strategies, and will moreover provide information about topologies that are not covered by the results in the database. These so-called "missing topologies" are further classified into asymmetric branch and long cascade decay topologies. While the decomposition procedure is model independent, the application of the simplified model results relies on a set of assumptions: the details of the production process are not important, only on-shell particles are

\footnotetext{
${ }^{1}$ An EM corresponds to a grid of simulated acceptance times efficiency values.
} 
relevant to the description, and BSM particles are sufficiently well described by their mass, ignoring all other quantum numbers. The validity of these assumptions should be tested before applying SModelS to a non-MSSM scenario.

\section{Application to the pMSSM}

A large reinterpretation study of the Run 1 ATLAS SUSY searches has been presented in [4], and we have used the results of that study to test the coverage of excluded points in the pMSSM by simplified model results [5]. Using SModelS we find that 55 (63) \% of Bino (Higgsino) LSP scenarios excluded by the ATLAS searches can also be excluded by the simplified model results. Figure 1 (left) shows the coverage for Bino LSP scenarios in the plane of gluino vs LSP mass. We see that light gluino scenarios are well covered except for the compressed region, but the coverage drops for intermediate gluino masses. The main reason is that cascade decays are often preferred and many topologies are not covered by the existing simplified model interpretations. One missing topology that was found to be particularly important arises from gluino-squark production, yielding a 3 jet $+E_{T}^{\text {miss }}$ topology. Note that the jets may be either quark or gluon jets, and this topology can therefore be important both if the squark is lighter or heavier than the gluino. Figure 1 (right) shows the weight of this missing topology for points passing all SModelS constraints, in the plane of gluino mass vs the lightest squark mass. Including this topology is thus expected to boost the coverage by simplified models.
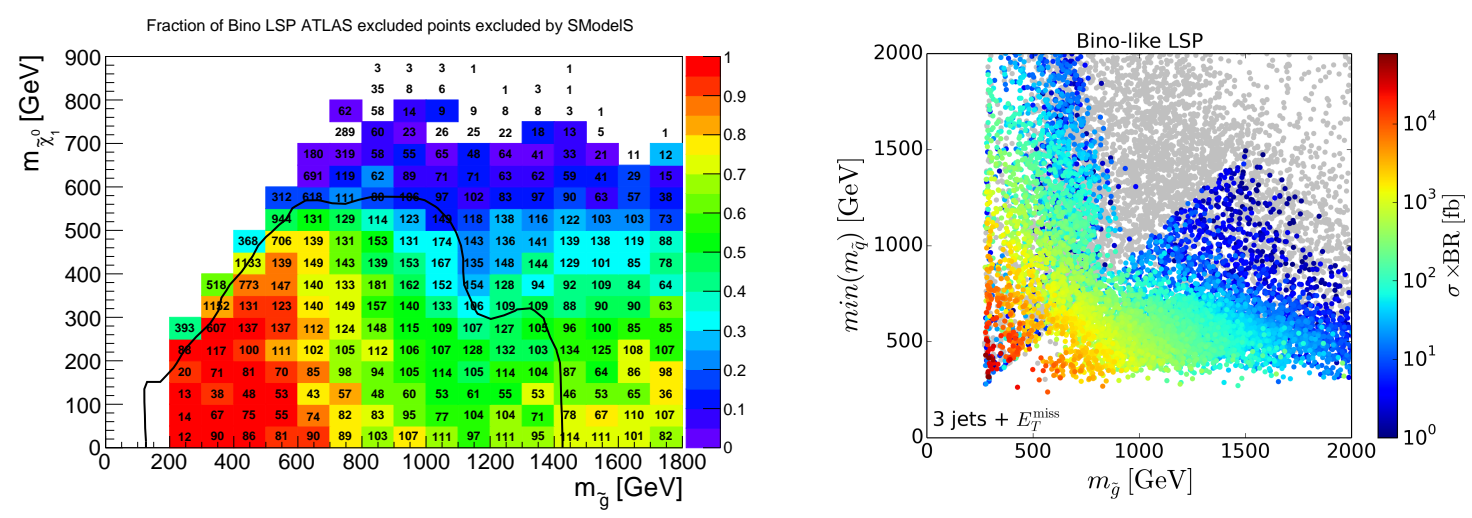

Figure 1: Left: coverage of the ATLAS excluded Bino LSP scenarios by SModelS, in the plane of gluino vs LSP mass. The black line indicates exclusion in the gluino direct decay simplified model from [6]. Right: 3 jet $+E_{T}^{\text {miss }}$ missing topology weight for SModelS allowed Bino LSP scenarios, in the gluino vs lightest squark mass plane.

\section{New Developments}

Several improvements have been included in recent releases. First, the database is continuously being updated, e.g. the inclusion of $13 \mathrm{TeV}$ results has been shown to have a big impact in excluding pMSSM scenarios [7]. In addition, major updates have become available with recent releases. Since version 1.1.3 the combination of signal regions is possible when covariance matrices are provided by the experiments, using the framework of simplified likelihoods [8]. Moreover, 
since version 1.2 the particle lifetime is used as an input to the decomposition, and constraints on simplified models with heavy stable charged particles or R-hadrons traversing the detector have become available [9].

\section{Conclusions and Outlook}

SModelS is a useful tool, both for fast tests against experimental results, and for more detailed studies of the collider phenomenology of models with a $\mathbb{Z}_{2}$ symmetry. Recent developments provide important additional functionalities and improved coverage. We expect to extend them further, both by extending the database and functionality. One example is the inclusion of efficiency maps for the important 3 jets $+E_{T}^{\text {miss }}$ topology. Another potential improvement is the treatment displaced vertex signatures which are not captured by the current description of long-lived particles.

\section{References}

[1] S. Kraml, S. Kulkarni, U. Laa, A. Lessa, W. Magerl, D. Proschofsky-Spindler et al., SModelS: a tool for interpreting simplified-model results from the LHC and its application to supersymmetry, Eur. Phys. J. C74 (2014) 2868 [1312 . 4175].

[2] S. Kraml, S. Kulkarni, U. Laa, A. Lessa, V. Magerl, W. Magerl et al., SModelS v1.0: a short user guide, 1412.1745 .

[3] F. Ambrogi, S. Kraml, S. Kulkarni, U. Laa, A. Lessa, V. Magerl et al., SModelS v1.1 user manual: Improving simplified model constraints with efficiency maps, Comput. Phys. Commun. 227 (2018) 72 [1701.06586].

[4] ATLAS collaboration, G. Aad et al., Summary of the ATLAS experiments sensitivity to supersymmetry after LHC Run 1 interpreted in the phenomenological MSSM, JHEP 10 (2015) 134 [1508.06608].

[5] F. Ambrogi, S. Kraml, S. Kulkarni, U. Laa, A. Lessa and W. Waltenberger, On the coverage of the pMSSM by simplified model results, Eur. Phys. J. C78 (2018) 215 [1707.09036].

[6] ATLAS collaboration, G. Aad et al., Search for squarks and gluinos with the ATLAS detector in final states with jets and missing transverse momentum using $\sqrt{s}=8 \mathrm{TeV}$ proton-proton collision data, JHEP 09 (2014) 176 [1405.7875].

[7] J. Dutta, S. Kraml, A. Lessa and W. Waltenberger, SModelS extension with the CMS supersymmetry search results from Run 2, LHEP 1 (2018) 5 [1803.02204].

[8] A. Buckley, M. Citron, S. Fichet, S. Kraml, W. Waltenberger and N. Wardle, The Simplified Likelihood Framework, 1809.05548.

[9] J. Heisig, S. Kraml and A. Lessa, Constraining new physics with searches for long-lived particles: Implementation into SModelS, 1808.05229. 